\title{
Pressure-Tuned Superconducting Dome in Chemically-Substituted $\kappa-(\mathrm{BEDT}-\mathrm{TTF})_{2} \mathrm{Cu}_{2}(\mathrm{CN})_{3}$
}

\author{
Yohei Saito ${ }^{1,+} \mathbb{C}$, Anja Löhle ${ }^{1,+}$, Atsushi Kawamoto $\left.{ }^{2} \mathbb{(}\right)$, Andrej Pustogow $1,3, * \mathbb{C}$ and Martin Dressel ${ }^{1, * \mathbb{C}}$ \\ 1 1. Physikalisches Institut, Universität Stuttgart, Pfaffenwaldring 57, 70569 Stuttgart, Germany; \\ yohei.saito@physik.uni-stuttgart.de (Y.S.); anjaloehle@googlemail.com (A.L.) \\ 2 Department of Physics, Graduate School of Science, Hokkaido University, Sapporo 060-0810, Japan; \\ atkawa@phys.sci.hokudai.ac.jp \\ 3 Institute of Solid State Physics, TU Wien, 1040 Vienna, Austria \\ * Correspondence: pustogow@ifp.tuwien.ac.at (A.P.); dressel@pi1.physik.uni-stuttgart.de (M.D.); \\ Tel.: +49-711-685-64946 (A.P. \& M.D.); Fax: +49-711-685-64886 (A.P. \& M.D.) \\ $\dagger$ These authors contributed equally.
}

Citation: Saito, Y.; Löhle, A.; Kawamoto, A.; Pustogow, A.; Dressel, M. Pressure-Tuned Superconducting Dome in Chemically-Substituted $\kappa-(\mathrm{BEDT}-\mathrm{TTF})_{2} \mathrm{Cu}_{2}(\mathrm{CN})_{3}$. Crystals 2021, 11, 817. https://doi.org/ $10.3390 /$ cryst 11070817

Academic Editor: Andrei Vladimirovich Shevelkov

Received: 31 May 2021

Accepted: 12 July 2021

Published: 14 July 2021

Publisher's Note: MDPI stays neutral with regard to jurisdictional claims in published maps and institutional affiliations.

Copyright: (c) 2021 by the authors. Licensee MDPI, Basel, Switzerland. This article is an open access article distributed under the terms and conditions of the Creative Commons Attribution (CC BY) license (https:// creativecommons.org/licenses/by/ $4.0 /)$.

\begin{abstract}
The quantum spin liquid candidate $\kappa-(B E D T-T T F)_{2} \mathrm{Cu}_{2}(\mathrm{CN})_{3}$ has been established as the prime example of a genuine Mott insulator that can be tuned across the first-order insulatormetal transition either by chemical substitution or by physical pressure. Here, we explore the superconducting state that occurs at low temperatures, when both methods are combined, i.e., when $\kappa$ $\left[(\mathrm{BEDT}-\mathrm{TTF})_{1-x}(\mathrm{BEDT}-\mathrm{STF})_{x}\right]_{2} \mathrm{Cu}_{2}(\mathrm{CN})_{3}$ is pressurized. We discovered superconductivity for partial BEDT-STF substitution with $x=0.10-0.12$ even at ambient pressure, i.e., a superconducting state is realized in the range between a metal and a Mott insulator without magnetic order. Furthermore, we observed the formation of a superconducting dome by pressurizing the substituted crystals; we assigned this novel behavior to disorder emanating from chemical tuning.
\end{abstract}

Keywords: charge-transfer salts; BEDT-TTF salts; pressure-dependent transport; metal-insulator transition; superconductivity

PACS: $71.30 .+\mathrm{h} ;$ 78.30.Jw; 75.25.Dk; 74.70.Kn

\section{Introduction}

Unconventional superconductivity remains one of the hot topics of condensed-matter research. Despite its discovery half a century ago and intense experimental scrutiny, the pairing mechanism remains an unsolved issue until today. Systematic investigations via controlled variation of distinct material parameters can shed new light on the phenomenon. Tuning via doping, pressure, and magnetic fields resulted in very similar phase diagrams in the cuprates, iron pnictides, alkali fullerides, and heavy fermion systems [1-10], all of which exhibit a superconducting dome between a Fermi liquid metal and an insulating or magnetically ordered state with the transition being suppressed on either side. Tuning $T_{\mathcal{C}}$ in small increments reveals intriguing scaling relations with the superconducting stiffness and the DC resistivity [11-14], which remain controversially discussed [15]. While in doped cuprates, imperfections and disorder are a serious issue [16-18], bandwidth tuningfor instance, by physical pressure-provides the opportunity to inspect stoichiometric compounds and assess the relevance of spatial inhomogeneity.

In this regard, organic superconductors serve as a versatile workbench because the bandwidth can be readily modified by hydrostatic pressure, by positive or negative strain, but also by chemical means via the exchange of anions or the variation of cations. Prime examples are the $\kappa$-phase BEDT-TTF salts [19-21]: in Figure $1 \mathrm{a}$, we see that right at the border between the magnetically ordered phase and Fermi liquid metal, superconductivity occurs at low temperatures. Other examples from rather diverse electron systems exhibit a 
very similar phenomenon, albeit the order parameters and energy scales might vastly differ [1-10,22,23]; nevertheless, a universal description of unconventional superconductivity in Fermionic systems is still elusive. One idea is that the occurrence of a superconducting phase around metal-insulator transitions and quantum critical points is related to the fact that the quasiparticles constituting a Fermi liquid are at the verge of destruction. Commonly, $T_{\mathcal{c}}$ is enhanced through an increase of the density of states at $E_{F}$ as correlations proliferate. On the other hand, the order parameter and its symmetry can be strongly affected by a change in spin fluctuations emerging from the insulating or magnetically ordered state nearby $[24,25]$.

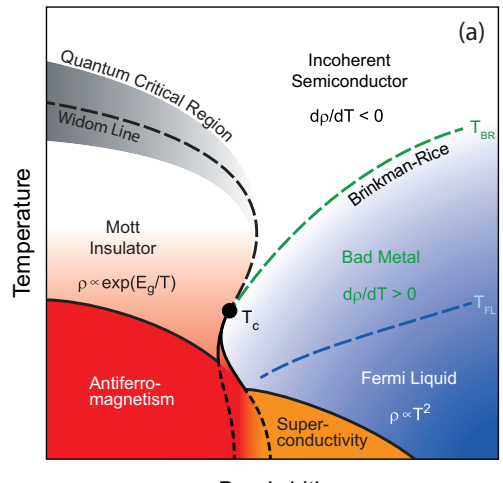

Bandwidth

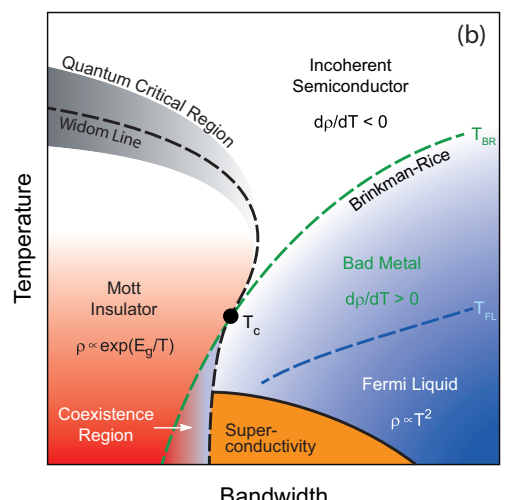

Bandwidth

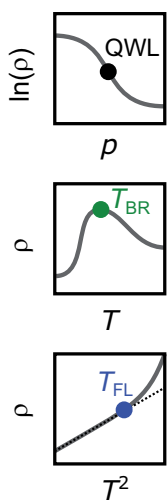

$T^{2}$

Figure 1. Schematic phase diagram of the quasi-two-dimensional $\kappa$-(BEDT-TTF) $)_{2} X$ salts as prime examples of bandwidth-tuned Mott insulators, where the effect of electronic correlations is reduced with increasing bandwidth. While at high temperatures, an incoherent semiconducting state is found for all systems, the behavior becomes distinct depending on the effect of electronic interactions. For strongly correlated compounds, a Mott-insulating behavior sets in when passing the quantum Widom line (QWL) upon cooling. Weakly correlated systems enter a bad metallic transport regime below the Brinkman-Rice temperature $T_{\mathrm{BR}}$, and Fermi-liquid-like $\rho(T) \propto T^{2}$ behavior occurs below $T_{\mathrm{FL}}$. At the verge of metal and insulator, a small region of superconductivity is found typically below 5 to $10 \mathrm{~K}$, but quickly suppressed. The right panels indicate the resistivity signatures of the crossovers in DC transport. The steepest slope of $\ln \{\rho(T)\}$ indicates the crossing of the quantum Widom line upon a pressure sweep. $T_{B R}$ indicates the crossover from an insulating $(\mathrm{d} \rho / \mathrm{d} T<0)$ to a metallic $(\mathrm{d} \rho / \mathrm{d} T>0)$ temperature dependence [26-28]. (a) In most cases, Mott insulators order antiferromagnetically, such as $\kappa-(\mathrm{BEDT}-\mathrm{TTF})_{2} \mathrm{Cu}\left[\mathrm{N}(\mathrm{CN})_{2}\right] \mathrm{Cl}$; by physical or chemical pressure, the systems undergo a transition from an antiferromagnetic insulator to the superconducting state, such as in $\kappa-(\mathrm{BEDT}-\mathrm{TTF})_{2} \mathrm{Cu}\left[\mathrm{N}(\mathrm{CN})_{2}\right] \mathrm{Br}$ or $\kappa-(\mathrm{BEDT}-\mathrm{TTF})_{2} \mathrm{Cu}(\mathrm{NCS})_{2}$, for instance. (b) Fully frustrated systems, such as $\kappa$-(BEDT-TTF $)_{2} \mathrm{Cu}_{2}(\mathrm{CN})_{3}$, remain paramagnetic down to the lowest temperatures, inferring a quantum spin liquid state. Here, a region of phase coexistence is entered by external pressure or chemical substitution. Eventually, a metallic or even superconducting state occurs. For more details, see the recent review [23].

The organic compounds are special, as they (i) realize unconventional superconductivity between phases that do not involve symmetry breaking (paramagnetic Mott insulator and Fermi liquid) and (ii) do not exhibit a superconducting dome, but instead, a continuously decreasing $T_{c}$ as correlations diminish (Figure 1). The quantum spin liquid candidate $\kappa$-(BEDT-TTF $)_{2} \mathrm{Cu}_{2}(\mathrm{CN})_{3}$ might be a suitable candidate to shed more light on this issue because no magnetic order occurs down to the lowest temperatures [29-32]; hence, a transition from a genuine Mott insulator to a Fermi liquid occurs, as depicted in Figure 1b. In a series of studies, this paradigmatic insulator-metal transition was thoroughly investigated during the last few years $[27,28,31,33-36]$, with particular emphasis on the transition from a correlated quantum spin liquid Mott insulator via a coexistence regime to a Fermi liquid metal. Here, we consider how the superconducting state evolves when the bandwidth of the system is tuned by chemical substitution and physical pressure simultaneously. 
The comparison of the two methods provides insight into the effects of disorder that are-similar to chemically doped materials—inevitable for partial substitution.

\section{Results}

\subsection{Experiments}

In 1991, Jack M. Williams and collaborators discovered [37] that the dimer Mott insulator $\kappa$-(BEDT-TTF $)_{2} \mathrm{Cu}_{2}(\mathrm{CN})_{3}$ becomes superconducting at around $T_{\mathcal{c}}=3-4 \mathrm{~K}$ when hydrostatic pressure above approximately $1.4 \mathrm{kbar}$ is applied; it took more than a decade before this observation was confirmed and finally followed up [30,31]. Although the required pressure is not as small as in the case of $\kappa$-(BEDT-TTF $)_{2} \mathrm{Cu}\left[\mathrm{N}(\mathrm{CN})_{2}\right] \mathrm{Cl}$, it is still lower than in $\kappa$-(BEDT-TTF $)_{2} \mathrm{Ag}_{2}(\mathrm{CN})_{3}$ or many other organic compounds; in other words, the title compound is located close to the metallic phase in the diagram sketched in Figure 1b. It is interesting to note that the superconducting transition happens at rather low temperatures, possibly a result of geometrical frustration of the exchange interactions, while it exceeds $10 \mathrm{~K}$ in the case of $\kappa$-(BEDT-TTF $)_{2} \mathrm{Cu}(\mathrm{NCS})_{2}$ or $\kappa$-(BEDT-TTF $)_{2} \mathrm{Cu}\left[\mathrm{N}(\mathrm{CN})_{2}\right] \mathrm{Br}$ [19-21], where antiferromagnetism occurs on the insulating side (cf. Figure 1a). Here, we want to take another approach to gradually increase the electronic bandwidth of $\kappa$-(BEDT-TTF $)_{2} \mathrm{Cu}_{2}(\mathrm{CN})_{3}$ : we replace some of the organic BEDT-TTF molecules by BEDT-STF molecules as illustrated in Figure 2 because selenium possesses larger orbitals than sulfur. This chemical substitution is well known from the quasi-one-dimensional TMTTF and TMTSF salts $[19,38,39]$.

(a)

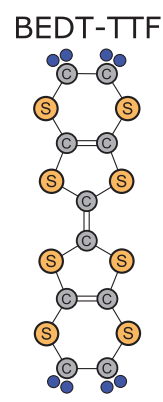

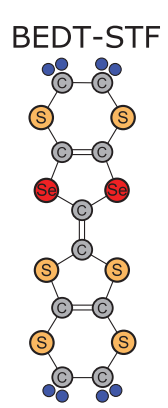

(b)

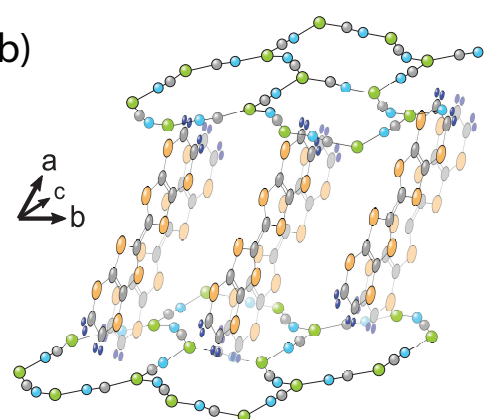

network of polymeric $\mathrm{Cu}_{2}(\mathrm{CN})_{3}$

dimers of BEDT-TTF and BEDT-STF molecules

Figure 2. (a) Organic donor molecules bis-(ethylenedithio)-tetrathiafulvalene (BEDT-TTF) and bis(ethylenedithio)-diseleniumdithiafulvalene (BEDT-STF). In the latter case, two sulfur atoms of the inner rings are replaced by selenium. (b) The crystal structure contains dimers of the donor molecules forming layers in the $b c$-plane, which are separated by the $\mathrm{Cu}_{2}(\mathrm{CN})_{3}$ anion sheets. The compounds therefore constitute prime examples of two-dimensional conductors and superconductors.

Single crystals of $\kappa$-[(BEDT-TTF $\left.)_{1-x}(\mathrm{BEDT}-\mathrm{STF})_{x}\right]_{2} \mathrm{Cu}_{2}(\mathrm{CN})_{3}$ with various stoichiometries were prepared by electrochemical oxidation following standard procedures [40]. For the alloying series, the amount of donor molecules, BEDT-TTF and BEDT-STF, was preselected; for each batch, the actual substitution value $x$ was determined aposteriori by energy-dispersive $\mathrm{X}$-ray spectroscopy: using $\kappa$-(BEDT-TTF $)_{2} \mathrm{Cu}_{2}(\mathrm{CN})_{3}$ and $\kappa$-(BEDT-STF $)_{2}$ $\mathrm{Cu}_{2}(\mathrm{CN})_{3}$ as a references, we compared the intensity of Se atoms to that of $\mathrm{S}$ atoms to calculate the actual fraction of BEDT-STF by linear interpolation $[36,41]$. Superconductivity was probed by magnetic susceptibility studies on polycrystalline samples employing the commercial Quantum Design SQUID. We recorded the DC resistivity using four-point measurements within the $b c$-plane; also, magnetoresistance was investigated in a custommade setup with the magnetic field aligned normal to the plane. All experiments were restricted to ${ }^{4} \mathrm{He}$ temperatures $(T>1.4 \mathrm{~K})$. The pressure-dependent electrical resistivity measurements were carried out using a clamp piston pressure cell [42] with Daphne oil 7373 as the pressure-transmitting medium $[43,44]$. While cooling, an InSb sensor monitored the pressure in situ; the actual low-temperature values are reported throughout. 


\subsection{Physical Pressure vs. Partial Chemical Substitution}

In order to compare the electronic properties of pressurized $\kappa$-(BEDT-TTF $)_{2} \mathrm{Cu}_{2}(\mathrm{CN})_{3}$ with the alloy series $\kappa-\left[(\text { BEDT-TTF })_{1-x}(\text { BEDT-STF })_{x}\right]_{2} \mathrm{Cu}_{2}(\mathrm{CN})_{3}$, an overview of the temperature-dependent resistivity is presented in Figure 3. The Mott insulator is characterized by an activated transport behavior that gradually weakens with pressure. For $p=1 \mathrm{kbar}$, the first traces of metallic conduction are seen below $50 \mathrm{~K}$, but $\rho(T)$ still remains two orders of magnitude above the room-temperature value, as seen in Figure 3a. Together with the nonmonotonic temperature dependence, this is a clear sign of metal-insulator phase separation in the coexistence region [34,35]. Only for $p \geq 1.2 \mathrm{kbar}$, we clearly observe metallic conductivity at low temperatures with a sharp onset of superconductivity at $T_{\mathrm{SC}}=4.8 \mathrm{~K}$; the midpoint is at $4 \mathrm{~K}$. The maximum resistivity identifies the Brinkman-Rice temperature $T_{\mathrm{BR}}[28,34]$. With rising pressure, it shifts to a higher temperature, as illustrated in Figure 1.
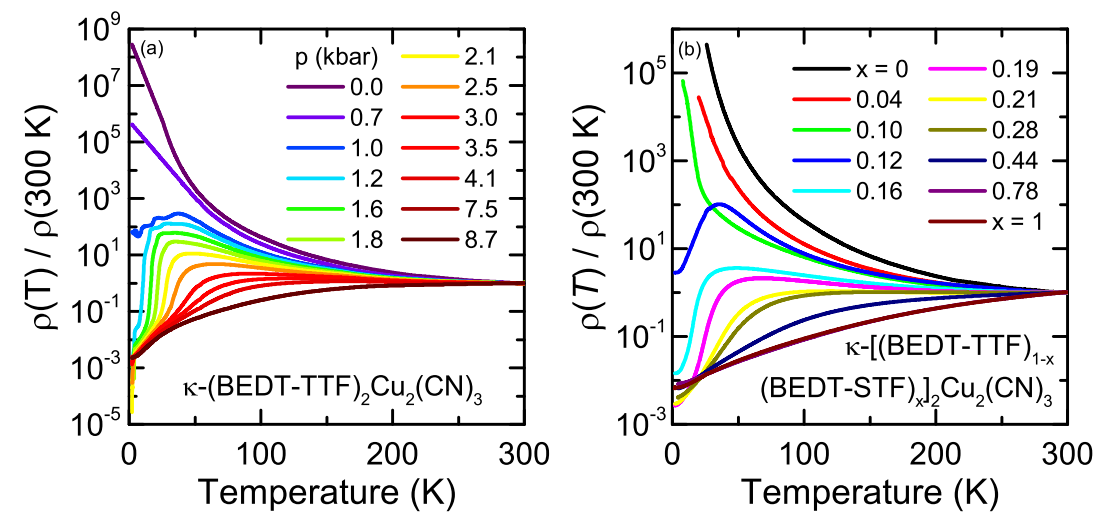

Figure 3. (a) Temperature-dependence of the DC resistivity of $\kappa$-(BEDT-TTF $)_{2} \mathrm{Cu}_{2}(\mathrm{CN})_{3}$ measured within the highly conducting $b c$-plane when applying hydrostatic pressure. The pressure drop upon cooling was monitored in situ; here, we indicate the values near $T=0$. (b) Temperature-dependent resistivity of $\kappa-\left[(\mathrm{BEDT}-\mathrm{TTF})_{1-x}(\mathrm{BEDT}-\mathrm{STF})_{x}\right]_{2} \mathrm{Cu}_{2}(\mathrm{CN})_{3}$ where, in the inner rings of the BEDT-TTF molecules, sulfur was partially substituted by selenium. $\rho(T)$ is normalized to room temperature.

From the resistivity data in Figure 3a, it is important to note that for pressure sufficient to enter the metallic state, superconductivity occurs immediately; in other words, the superconducting phase is present next to the Mott insulator, with a regime of phase coexistence, as illustrated in Figure 1b. The distinct superconducting transition for $p=1.2 \mathrm{kbar}$ progressively shifts to lower temperatures and becomes more gradual, in line with the early report by Geiser et al. [37]. For pressures above $4 \mathrm{kbar}$, superconducting transition can no longer be identified in the accessible temperature range; this is in good agreement with previous work [30].

If we now turn to the substitutional series $\kappa-\left[(\mathrm{BEDT}-\mathrm{TTF})_{1-x}(\mathrm{BEDT}-\mathrm{STF})_{x}\right]_{2} \mathrm{Cu}_{2}(\mathrm{CN})_{3}$ presented in Figure 3b, a similar development is found as the BEDT-STF content $x$ increases. While for $x<0.1$, the Mott insulator exhibits a simple activated behavior, the increase of resistivity slows down markedly for $x=0.10$ at $T<14 \mathrm{~K}$, indicating that the coexistence regime is entered with a metallic volume fraction smaller than the percolation threshold. Fully metallic behavior is observed for the $x=0.12$ sample below the pronounced resistivity maximum at approximately $35 \mathrm{~K}$. However, $\rho(T)$ saturates at low temperatures without becoming a good metal; we assigned the large resistivity values above the Ioffe-Regel-Mott limit to metal-insulator coexistence [34,36]. Only when $x$ rises further, a regular Fermi liquid behavior is seen below the Brinkman-Rice temperature $T_{\mathrm{BR}}$, as discussed in full detail in [28]. From our simple DC measurements at $p=0$, we cannot unambiguously identify bulk superconductivity in any of the crystals down to $T \approx 1.4 \mathrm{~K}$. Note that, due to the high resistance, the data for $x=0.00,0.04$, and 0.10 are limited to $T>26,20$, and $8 \mathrm{~K}$, respectively. 


\subsection{Ambient-Pressure Superconductivity between Spin Liquid and Fermi Liquid}

Since magnetic susceptibility is a more sensitive method to detect traces of superconductivity, we performed SQUID measurements on several $\kappa$-[(BEDT-TTF $)_{1-x}$ (BEDT$\left.\mathrm{STF})_{x}\right]_{2} \mathrm{Cu}_{2}(\mathrm{CN})_{3}$ single crystals at ambient pressure. To that end, the samples were cooled down in the absence of an external field (zero-field cooled (ZFC)), and subsequently, the data were recorded during warming up, applying a weak field of $B=0.01 \mathrm{~T}$. In Figure $4 \mathrm{a}$, we detect a clear drop in the relative magnetic susceptibility $\Delta \chi_{S}(T)$ that evidences traces of superconductivity for the compositions $x=0.10$ and $x=0.12$. Unfortunately, we cannot follow the behavior to lower temperatures in order to estimate the background and superconducting volume fraction reliably. Nevertheless, we can determine $T_{\mathcal{c}}=(2.7 \pm 0.2) \mathrm{K}$ for $x=0.12$ and $T_{c}=(3.0 \pm 0.2) \mathrm{K}$ for $x=0.10$ as the temperature where significant deviations occur. As seen from the $p=0.0$ kbar curve in Figure $5 c$, the low-temperature resistivity of the composition $x=0.12$ exhibits a small, but noticeable drop below approximately $2.6 \mathrm{~K}$, in agreement with the SQUID result in Figure 4a.

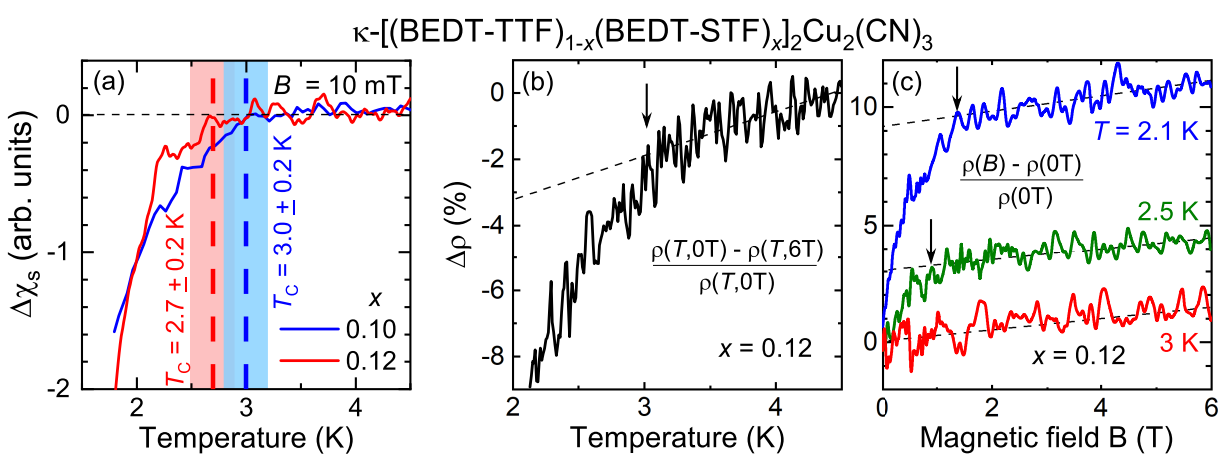

Figure 4. (a) Temperature-dependent magnetic susceptibility of polycrystalline $\kappa$-[(BEDT-TTF) $1-x^{-}$ $\left.(\text { BEDT-STF })_{x}\right]_{2} \mathrm{Cu}_{2}(\mathrm{CN})_{3}$ for the substitution values $x=0.10$ and 0.12 measured by a SQUID in an external field of $10 \mathrm{mT}$. The superconducting transition $T_{\mathcal{C}}=2.7 \mathrm{~K}$ and $3.0 \mathrm{~K}$ can be determined from the temperature where $\chi_{S}(T)$ starts to drop. The data were taken in zero-field-cooled mode upon warming up. (b) Normalized DC resistivity of $\kappa$-[(BEDT-TTF $\left.)_{0.88}(\text { BEDT-STF })_{0.12}\right]_{2} \mathrm{Cu}_{2}(\mathrm{CN})_{3}$ comparing a zero magnetic field with $B=6 \mathrm{~T}$. The strong deviation below $3 \mathrm{~K}$ indicates the suppression of superconductivity. (c) The magnetoresistance $\Delta \rho$ corresponds to the relative change in resistivity due to an external magnetic field $B$. While for higher temperatures $(T=3 \mathrm{~K})$, there is linear increase of $\Delta \rho(B)$ with $B$, a kink is observed at lower temperatures, indicated by the black arrows. This corresponds to the suppression of the superconductivity, i.e., the upper critical field.
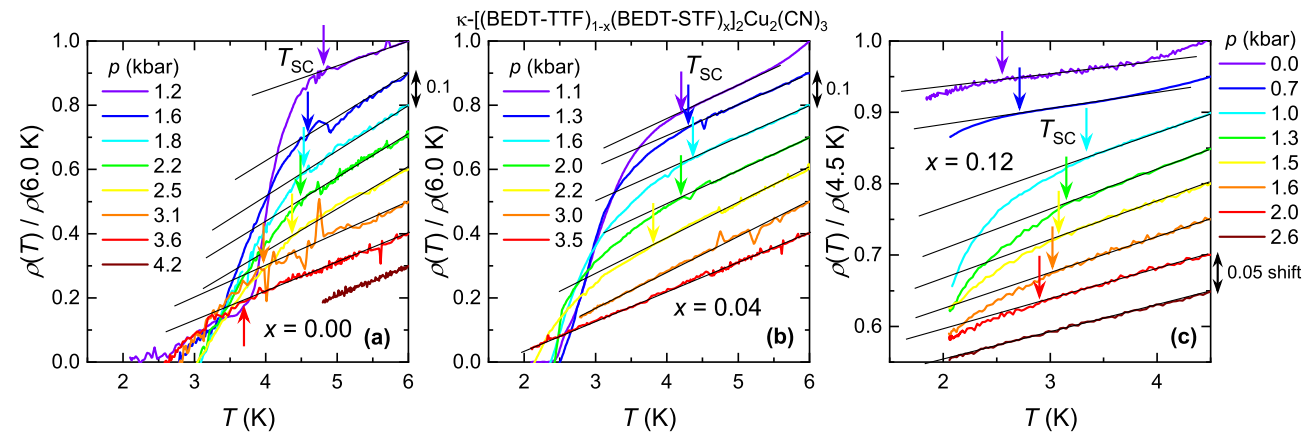

Figure 5. (a) Normalized electrical resistivity $\rho(T) / \rho(6.5 \mathrm{~K})$ of $\kappa$-(BEDT-TTF $)_{2} \mathrm{Cu}_{2}(\mathrm{CN})_{3}$ plotted as a function of temperature for different values of hydrostatic pressure applied, ranging from 1.2 to 4.2 kbar. The transition temperature gradually decreases for $p>1.2 \mathrm{kbar}$. (b) For $x=0.04$, $\rho(T) / \rho(6.5 \mathrm{~K})$ reveals an initial increase of $T_{S C}$, followed by a reduction until it is fully suppressed. (c) The dome-like increase of transition temperature with pressure is even more pronounced for $x=0.12(\rho(T)$ normalized to $4.5 \mathrm{~K})$. For clarity, the curves in all panels are shifted vertically with respect to each other. 
In addition, we confirmed the superconducting state by magnetotransport measurements at ambient pressure. For this, the data taken at zero field were directly compared with those recorded in the presence of a strong external magnetic field. In Figure $4 \mathrm{~b}$, we plot the according changes in resistivity for $\kappa-\left[(\mathrm{BEDT}-\mathrm{TTF})_{1-x}(\mathrm{BEDT}-\mathrm{STF})_{x}\right]_{2} \mathrm{Cu}_{2}(\mathrm{CN})_{3}$ with $x=0.12$. When an external magnetic field of $B=6 \mathrm{~T}$ is applied, a kink occurs for temperatures below $3 \mathrm{~K}$, slightly higher, but in overall agreement with the SQUID result $\left(T_{c}=(2.7 \pm 0.2) \mathrm{K}\right)$, indicating the destruction of the superconducting phase. The fielddependent change in resistivity $\Delta \rho$ by an external magnetic field $B$ is presented in Figure $4 c$ for three different temperatures $T=2.1,2.5$, and $3 \mathrm{~K}$. For the lower temperatures, a kink around 1 and $1.4 \mathrm{~T}$ indicates the upper critical field. Unfortunately, the quality of the data does not allow a more reliable determination. We suspect that the composition $x=0.12$ involves only a small volume fraction of (super)conducting regions as it is located in the coexistence regime with metallic puddles in an insulating matrix $[34,36]$. For $x=0.10$, where the resistivity data from Figure $3 \mathrm{~b}$ indicated no metallic transport down to $8 \mathrm{~K}$, the (super)conducting volume fraction is likely even smaller than the percolation threshold.

\subsection{Pressure Evolution Reveals a Superconducting Dome in Chemically Substituted Samples}

Having discovered traces of superconductivity in the $x=0.10$ and 0.12 samples of $\kappa$-[(BEDT-TTF $\left.)_{1-x}(\text { BEDT-STF })_{x}\right]_{2} \mathrm{Cu}_{2}(\mathrm{CN})_{3}$, we investigated the pressure evolution of this state in more detail. Figure 5 displays the temperature-dependent resistivity $\rho(T)$ of $x=0.00$ (parent compound), 0.04 , and 0.12 normalized to the $T=6 \mathrm{~K}$ and $4.5 \mathrm{~K}$ value, respectively. Note that, to ensure a consistent comparison, we determined the transition temperature as the onset of superconductivity, i.e., the drop of $\rho(T)$ defined as the change in curvature turning from concave to convex, as indicated by the vertical arrows. Since this temperature is larger than the values reported in the literature, where the steepest slope is the most common criterion, here we use the notation $T_{S C}$ instead of $T_{c}$. When a hydrostatic pressure of $1 \mathrm{kbar}$ is applied, $\kappa$-[(BEDT-TTF $\left.)_{0.96}(\mathrm{BEDT}-\mathrm{STF})_{0.04}\right]_{2} \mathrm{Cu}_{2}(\mathrm{CN})_{3}$ becomes superconducting at $T_{S C}=4.2 \mathrm{~K}$; increasing the pressure further, the transition temperature rises up to $4.4 \mathrm{~K}$ for $p=1.6 \mathrm{kbar}$ and decreases above that. A similar behavior is observed in $\kappa$-[(BEDT-TTF $\left.)_{0.88}(\mathrm{BEDT}-\mathrm{STF})_{0.12}\right]_{2} \mathrm{Cu}_{2}(\mathrm{CN})_{3}$. While for ambient pressure, the critical temperature is around $2.6 \mathrm{~K}$, slightly lower than the magnetization and magnetotransport results, we discovered a dome with a maximum of almost $3.4 \mathrm{~K}$ at $p=1 \mathrm{kbar}$ before $T_{S C}$ drops again. The transition temperatures of the three investigated compounds ( $x=0.00,0.04$, and 0.12) are summarized in Figure 6.

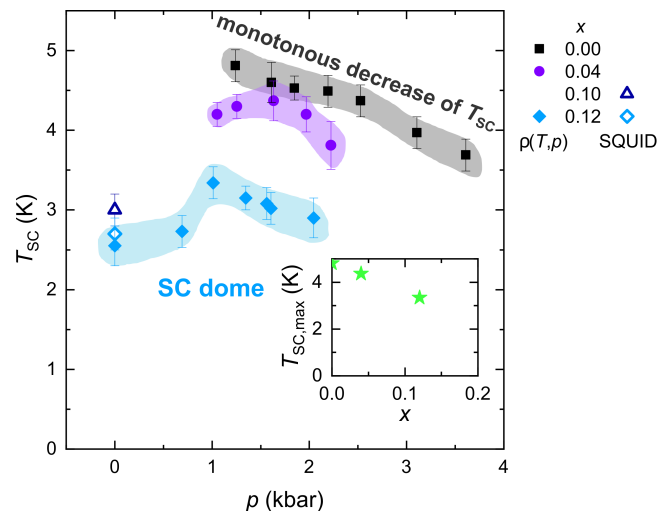

Figure 6. Superconducting transition temperature $T_{S C}$ for $\kappa-\left[(B E D T-T T F)_{1-x}(B E D T-S T F)_{x}\right]_{2} \mathrm{Cu}_{2}(C N)_{3}$ with $x=0.00,0.04$, and 0.12 as a function of pressure. In addition to the results from DC transport $\rho(T, p)$ (solid symbols; see Figure 5), the open symbols indicate the ambient pressure magnetization data points at $x=0.10$ and $x=0.12$ (see Figure 4 ). While the parent compound $(x=0.00)$ exhibits a monotonous suppression of the superconducting transition temperature with $p$, a dome-like structure develops for $x=0.04$ and 0.12 . The shaded areas following the trend of $T_{S C}$ in the respective colors are guides to the eye. The inset illustrates the reduction of the maximum value of $T_{S C}$ upon chemical substitution $x$. 


\section{Discussion}

Although the topic of a superconducting dome became popular only with hightemperature superconductors, such as cuprates and iron pnictides $[4,16,45,46]$, the first appearance dates back to the late 1960s in granular aluminum [47-49], where it is still under active research [50]. It was demonstrated that $T_{\mathcal{c}}$ is enhanced as the quantum confinement is strengthened, but phase fluctuations across the grain array become stronger and eventually suppress $T_{\mathcal{C}}$. The dispute about $\mathrm{Nb}$-doped $\mathrm{SrTiO}_{3}$ has a similarly long history [51-56]. Recently, a superconducting dome was observed in gate-tuned semiconducting transition metal dichalcogenides and twisted bilayer graphene [57-59]. However, also heavy fermion compounds, such as $\mathrm{CeIn}_{3}$ or $\mathrm{CePd}_{2} \mathrm{Si}_{2}$, exhibit superconducting domes when the system is pressurized [1]. Even in doped fullerides, which were recently identified as unconventional superconductors nearby a Mott transition [60], the transition temperature $T_{\mathcal{c}}$ goes through a maximum upon changing the lattice constant via chemical or physical pressure [60-62].

For all $\kappa$-phase BEDT-TTF compounds, there seems to be only a reduction of $T_{\mathcal{C}}$ with pressure, as depicted in Figure 1. To our knowledge, there is no report of a domelike behavior of $T_{\mathcal{C}}$ with pressure in any pristine charge-transfer salt [19-21,63]. The absence of a superconducting dome in clean systems is confirmed by our present study of $\kappa$-(BEDT-TTF $)_{2} \mathrm{Cu}_{2}(\mathrm{CN})_{3}$ under pressure, presented in Figure $5 \mathrm{a}$. When $p$ increases, there is an abrupt onset of superconductivity with $T_{S C}=4.8 \mathrm{~K}$, which then decreases continuously until the superconducting phase is completely suppressed.

In our substitutional series $\kappa$-[(BEDT-TTF $\left.)_{1-x}(\text { BEDT-STF })_{x}\right]_{2} \mathrm{Cu}_{2}(\mathrm{CN})_{3}$ for $x=0.10$ and 0.12 , we could realize- for the first time-ambient-pressure unconventional superconductivity between a Fermi liquid metal and a frustrated Mott insulator without magnetic order. Surprisingly, in the case of the two compounds $\kappa$-[(BEDT-TTF $)_{0.96}$ (BEDTSTF $\left.)_{0.04}\right]_{2} \mathrm{Cu}_{2}(\mathrm{CN})_{3}$ and $\kappa$ - $\left[(\mathrm{BEDT}-\mathrm{TTF})_{0.88}(\mathrm{BEDT}-\mathrm{STF})_{0.12} \mathrm{l}_{2} \mathrm{Cu}_{2}(\mathrm{CN})_{3}\right.$, we found a significant increase of $T_{S C}$ as pressure rises until it reaches a maximum and drops again (Figure 6). With larger substitution, the dome becomes more pronounced accompanied by a reduction of the maximum $T_{S C}$, as shown in the inset of Figure 6. Since the BEDT-STF molecules are randomly distributed within the BEDT-TTF layers, increasing their content also enhances disorder in the crystal [41]. To that end, we assigned the qualitative changes in the superconducting properties from a monotonous reduction towards a dome-like pressure dependence to disorder effects.

It comes at no surprise that spurious superconductivity with a reduction of $T_{C}$ on the insulating side was also reported in X-ray-irradiated $\kappa$-(BEDT-TTF $)_{2} \mathrm{Cu}\left[\mathrm{N}(\mathrm{CN})_{2}\right] \mathrm{Cl}[64]$, where disorder was increased on purpose. However, the amounts of randomness introduced into the system in that work and previous studies [65] were large enough to severely suppress antiferromagnetism and even affect the metal-insulator transition as a whole. In $\kappa$-[(BEDT-TTF $\left.)_{1-x}(\mathrm{BEDT}-\mathrm{STF})_{x}\right]_{2} \mathrm{Cu}_{2}(\mathrm{CN})_{3}$, on the other hand, the phase boundaries $\left(\mathrm{QWL}, T_{\mathrm{BR}}, T_{\mathrm{FL}}\right)$ remain rather unaffected by BEDT-STF substitution, yielding a conversion factor between pressure and substitution of $\Delta x / \Delta p \approx 0.055 \mathrm{kbar}^{-1}$ [28], and first-order phase coexistence was independently evidenced from the colossal percolative enhancement of the dielectric permittivity $\varepsilon_{1}[34,36]$. Clearly, superconductivity is more sensitive to disorder than the Mott(-Anderson) metal-insulator transition, because Cooper pair formation requires phase coherence. Hence, a similar scenario of inhomogeneous localization and percolation may be at work, as recently suggested for cuprates [17,18] — not only in the present case of organic Mott insulators, but also in fullerides and other unconventional superconductors exhibiting a dome of $T_{\mathcal{C}}$.

Our approach of partial chemical substitution enables systematic scrutiny of the superconducting state in organic charge-transfer salts. The ability to stabilize superconductivity at ambient pressure significantly improves experimental accessibility and reproducibility. When combining this tuning method with physical pressure, we obtained unprecedented control of the electronic properties. This way, we can disentangle the effects of bandwidth and disorder on the metal-insulator transition and Cooper pairing. Our finding of a superconducting dome not only puts the layered organic materials on par with other 
unconventional superconductors (cuprates, pnictides, fullerides, heavy fermions), but the absence of a dome in pristine charge-transfer salts unmasks them as the only clean realization of unconventional superconductivity at a metal-insulator transition.

\section{Conclusions}

Combining substitutional and pressure tuning of $\kappa-(\mathrm{BEDT}-\mathrm{TTF})_{2} \mathrm{Cu}_{2}(\mathrm{CN})_{3}$, we discovered novel ambient pressure superconductors $\kappa-\left[(\mathrm{BEDT}-\mathrm{TTF})_{1-x}(\mathrm{BEDT}-\mathrm{STF})_{x}\right]_{2} \mathrm{Cu}_{2}(\mathrm{CN})_{3}$, with $x$ between 0.10 and 0.12 . The transition temperature increases when pressure is applied, forming a superconducting dome, common to other unconventional superconductors, but rarely observed in organics. The dome diminishes and eventually vanishes as $x$ increases. We suggest the importance of disorder in this regard.

Author Contributions: Conceptualization and supervision, M.D.; crystal growth Y.S. and A.K.; investigation and analysis, A.L., A.P. and Y.S.; writing and editing, A.P. and M.D. All authors read and agreed to the published version of the manuscript.

Funding: Deutsche Forschungsgemeinschaft.

Institutional Review Board Statement: Not applicable.

Informed Consent Statement: Not applicable.

Data Availability Statement: Data are available from the corresponding author (M.D.) upon request.

Acknowledgments: The project was supported by the Deutsche Forschungsgemeinschaft (DFG) via DR228/39-3 and DR228/52-1.

Conflicts of Interest: The authors declare no conflict of interest.

\section{References}

1. Mathur, N.D.; Grosche, F.M.; Julian, S.R.; Walker, I.R.; Freye, D.M.; Haselwimmer, R.K.W.; Lonzarich, G.G. Magnetically mediated superconductivity in heavy fermion compounds. Nature 1998, 394, 39-43. [CrossRef]

2. Saxena, S.S.; Agarwal, K.A.P.; Grosche, F.M.; Haselwimmer, R.K.W.; Steiner, M.J.; Pugh, E.; Walker, I.R.; Julian, S.R.; Monthoux, P.; Lonzarich, G.G.; et al. Superconductivity on the border of itinerant-electron ferromagnetism in $\mathrm{UGe}_{2}$. Nature 2000, 406, 587-592. [CrossRef]

3. Löhneysen, H.V.; Rosch, A.; Vojta, M.; Wölfle, P. Fermi liquid instabilities at magnetic quantum phase transitions. Rev. Mod. Phys. 2007, 79, 1015-1075. [CrossRef]

4. Broun, D.M. What lies beneath the dome? Nat. Phys. 2008, 4, 170-172. [CrossRef]

5. Sachdev, S. Where is the quantum critical point in the cuprate superconductors? Phys. Stat. Sol. B 2010, 247, 537-543. [CrossRef]

6. Sachdev, S.; Keimer, B. Quantum criticality. Phys. Today 2011, 64, 29. [CrossRef]

7. Norman, M.R. The Challenge of Unconventional Superconductivity. Science 2011, 332, 196-200. [CrossRef]

8. Chapman, S.; Hoyos, C.; Oz, Y. Lifshitz superfluid hydrodynamics. J. High Energy Phys. 2014, 2014, 27, [CrossRef]

9. Keimer, B.; Kivelson, S.A.; Norman, M.R.; Uchida, S.; Zaanen, J. From quantum matter to high-temperature superconductivity in copper oxides. Nature 2015, 518, 179-186. [CrossRef]

10. Metlitski, M.A.; Mross, D.F.; Sachdev, S.; Senthil, T. Cooper pairing in non-Fermi liquids. Phys. Rev. B 2015, 91, 115111. [CrossRef]

11. Uemura, Y.J.; Luke, G.M.; Sternlieb, B.J.; Brewer, J.H.; Carolan, J.F.; Hardy, W.N.; Kadono, R.; Kempton, J.R.; Kiefl, R.F.; Kreitzman, S.R.; et al. Universal Correlations between $T_{\mathcal{C}}$ and $n_{s} / m^{*}$ (Carrier Density over Effective Mass) in High- $T_{\mathcal{C}}$ Cuprate Superconductors. Phys. Rev. Lett. 1989, 62, 2317-2320. [CrossRef]

12. Basov, D.N.; Timusk, T.; Dabrowski, B.; Jorgensen, J.D. $c$-axis response of $\mathrm{YBa}_{2} \mathrm{Cu}_{4} \mathrm{O}_{8}$ : A pseudogap and possibility of Josephson coupling of $\mathrm{CuO}_{2}$ planes. Phys. Rev. B 1994, 50, 3511-3514. [CrossRef] [PubMed]

13. Homes, C.C.; Dordevic, S.V.; Strongin, M.; Bonn, D.A.; Liang, R.; Hardy, W.N.; Komiya, S.; Ando, Y.; Yu, G.; Kaneko, N.; et al. A universal scaling relation in high-temperature superconductors. Nature 2004, 430, 539-541. [CrossRef] [PubMed]

14. Dordevic, S.V.; Basov, D.N.; Homes, C.C. Do organic and other exotic superconductors fail universal scaling relations? Sci. Rep. 2013, 3, 1713. [CrossRef]

15. Božović, I.; He, X.; Wu, J.; Bollinger, A.T. Dependence of the critical temperature in overdoped copper oxides on superfluid density. Nature 2016, 536, 309-311. [CrossRef] [PubMed]

16. Lee, P.A.; Nagaosa, N.; Wen, X.G. Doping a Mott insulator: Physics of high-temperature superconductivity. Rev. Mod. Phys. 2006, 78, 17-85. [CrossRef]

17. Pelc, D.; Vučković, M.; Grbić, M.S.; Požek, M.; Yu, G.; Sasagawa, T.; Greven, M.; Barišić, N. Emergence of superconductivity in the cuprates via a universal percolation process. Nat. Commun. 2018, 9, 4327. [CrossRef] 
18. Pelc, D.; Popčević, P.; Požek, M.; Greven, M.; Barišić, N. Unusual behavior of cuprates explained by heterogeneous charge localization. Sci. Adv. 2019, 5, eaau4538. [CrossRef]

19. Ishiguro, T.; Yamaji, K.; Saito, G. Organic Superconductors, 2nd ed.; Springer: Berlin, Germany, 1998.

20. Toyota, N.; Lang, M.; Müller, J. Low-Dimensional Molecular Metals; Springer Series in Solid-State Sciences; Springer: Berlin, Germany, 2007; Volume 154.

21. Lebed, A. (Ed.) The Physics of Organic Superconductors and Conductors; Springer Series in Materials Science; Springer: Berlin, Germany, 2008; Volume 110.

22. Dressel, M. Quantum criticality in organic conductors? Fermi liquid versus non-Fermi liquid behaviour. J. Phys. Condens. Matter 2011, 23, 293201. [CrossRef]

23. Dressel, M.; Tomić, S. Molecular quantum materials: Electronic phases and charge dynamics in two-dimensional organic solids. Adv. Phys. 2020, 69, 1-120. [CrossRef]

24. Limelette, P.; Wzietek, P.; Florens, S.; Georges, A.; Costi, T.A.; Pasquier, C.; Jérome, D.; Mézière, C.; Batail, P. Mott Transition and Transport Crossovers in the Organic Compound $\kappa-(\mathrm{BEDT}-\mathrm{TTF})_{2} \mathrm{Cu}\left[\mathrm{N}(\mathrm{CN})_{2}\right] \mathrm{Cl}$. Phys. Rev. Lett. 2003, $91,016401$. [CrossRef] [PubMed]

25. Lefebvre, S.; Wzietek, P.; Brown, S.; Bourbonnais, C.; Jérome, D.; Mézière, C.; Fourmigué, M.; Batail, P. Mott Transition, Antiferromagnetism, and Unconventional Superconductivity in Layered Organic Superconductors. Phys. Rev. Lett. 2000, 85, 5420-5423. [CrossRef] [PubMed]

26. Furukawa, T.; Miyagawa, K.; Taniguchi, H.; Kato, R.; Kanoda, K. Quantum criticality of Mott transition in organic materials. Nat. Phys. 2015, 11, 221-224. [CrossRef]

27. Pustogow, A.; Bories, M.; Löhle, A.; Rösslhuber, R.; Zhukova, E.; Gorshunov, B.; Tomić, S.; Schlueter, J.A.; Hübner, R.; Hiramatsu, T.; et al. Quantum spin liquids unveil the genuine Mott state. Nat. Mater. 2018, 17, 773-777. [CrossRef] [PubMed]

28. Pustogow, A.; Saito, Y.; Löhle, A.; Sanz Alonso, M.; Kawamoto, A.; Dobrosavljević, V.; Dressel, M.; Fratini, S. Rise and fall of Landau's quasiparticles while approaching the Mott transition. Nat. Commun. 2021, 12, 1571. [CrossRef] [PubMed]

29. Shimizu, Y.; Miyagawa, K.; Kanoda, K.; Maesato, M.; Saito, G. Spin Liquid State in an Organic Mott Insulator with a Triangular Lattice. Phys. Rev. Lett. 2003, 91, 107001. [CrossRef] [PubMed]

30. Kurosaki, Y.; Shimizu, Y.; Miyagawa, K.; Kanoda, K.; Saito, G. Mott Transition from a Spin Liquid to a Fermi Liquid in the Spin-Frustrated Organic Conductor $\kappa-(\mathrm{ET})_{2} \mathrm{Cu}_{2}(\mathrm{CN})_{3}$. Phys. Rev. Lett. 2005, 95. [CrossRef]

31. Furukawa, T.; Kobashi, K.; Kurosaki, Y.; Miyagawa, K.; Kanoda, K. Quasi-continuous transition from a Fermi liquid to a spin liquid in $\kappa-(\mathrm{ET})_{2} \mathrm{Cu}_{2}(\mathrm{CN})_{3}$. Nat. Commun. 2018, 9, 1-7. [CrossRef]

32. Miksch, B.; Pustogow, A.; Javaheri Rahim, M.; Bardin, A.A.; Kanoda, K.; Schlueter, J.A.; Hübner, R.; Scheffler, M.; Dressel, M. Gapped magnetic ground state in quantum spin-liquid candidate $\kappa$-(BEDT-TTF $)_{2} \mathrm{Cu}_{2}(\mathrm{CN})_{3}$. Science 2021, 372, $276-279$. [CrossRef] [PubMed]

33. Li, W.; Pustogow, A.; Kato, R.; Dressel, M. Transition of a pristine Mott insulator to a correlated Fermi liquid: Pressure-dependent optical investigations of a quantum spin liquid. Phys. Rev. B 2019, 99, 115137. [CrossRef]

34. Pustogow, A.; Rösslhuber, R.; Tan, Y.; Uykur, E.; Böhme, A.; Wenzel, M.; Saito, Y.; Löhle, A.; Hübner, R.; Kawamoto, A.; et al. Low-temperature dielectric anomalies arising from electronic phase separation at the Mott insulator-metal transition. NPJ Quantum Mater. 2021, 6, 9. [CrossRef]

35. Rösslhuber, R.; Pustogow, A.; Uykur, E.; Böhme, A.; Löhle, A.; Hübner, R.; Schlueter, J.A.; Tan, Y.; Dobrosavljević, V.; Dressel, M. Phase coexistence at the first-order Mott-transition revealed by pressure-dependent dielectric spectroscopy of $\kappa$-(BEDTTTF $)_{2} \mathrm{Cu}_{2}(\mathrm{CN})_{3}$. Phys. Rev. B 2021, 103, 125111. [CrossRef]

36. Saito, Y.; Rösslhuber, R.; Löhle, A.; Alonso, M.S.; Pustogow, A.; Kawamoto, A.; Dressel, M. Chemical tuning of molecular quantum materials $\kappa$-[(BEDT-TTF $\left.)_{1-x}(\text { BEDT-STF })_{x}\right]_{2} \mathrm{Cu}_{2}(\mathrm{CN})_{3}$ : From the Mott-insulating quantum spin liquid to metallic Fermi liquid. J. Mater. Chem. C 2021, 9. [CrossRef]

37. Geiser, U.; Wang, H.H.; Carlson, K.D.; Williams, J.M.; Charlier, H.A.; Heindl, J.E.; Yaconi, G.A.; Love, B.J.; Lathrop, M.W.; Schirber, J.E.; et al. Superconductivity at $2.8 \mathrm{~K}$ and $1.5 \mathrm{kbar}$ in $\kappa-(\mathrm{BEDT}-\mathrm{TTF})_{2} \mathrm{Cu}_{2}(\mathrm{CN})_{3}$ : The first organic superconductor containing a polymeric copper cyanide anion. Inorg. Chem. 1991, 30, 2586-2588. [CrossRef]

38. Jérome, D.; Schulz, H.J. Organic Conductors and Superconductors. Adv. Phys. 1982, 31, 299-490. [CrossRef]

39. Dressel, M. Spin-Charge Separation in Quasi One-Dimensional Organic Conductors. Naturwissenschaften 2003, 90, 337-344. [CrossRef] [PubMed]

40. Geiser, U.; Schultz, A.; Wang, H.; Watkins, D.; Stupka, D.; Williams, J.; Schirber, J.; Overmyer, D.; Jung, D.; Novoa, J.; et al. Strain index, lattice softness and superconductivity of organic donor-molecule salts: Crystal and electronic structures of three isostructural salts $\kappa$-(BEDT- TTF $)_{2} \mathrm{Cu}\left[\mathrm{N}(\mathrm{CN})_{2}\right] X(X=\mathrm{Cl}, \mathrm{Br}, \mathrm{I})$. Physica C 1991, 174, 475-486. [CrossRef]

41. Saito, Y.; Minamidate, T.; Kawamoto, A.; Matsunaga, N.; Nomura, K. Site-specific ${ }^{13} \mathrm{C}$ NMR study on the locally distorted triangular lattice of the organic conductor $\kappa-(\mathrm{BEDT}-\mathrm{TTF})_{2} \mathrm{Cu}_{2}(\mathrm{CN})_{3}$. Phys. Rev. B 2018, 98. [CrossRef]

42. Löhle, A.; Rose, E.; Singh, S.; Beyer, R.; Tafra, E.; Ivek, T.; Zhilyaeva, E.I.; Lyubovskaya, R.N.; Dressel, M. Pressure dependence of the metal-insulator transition in $\kappa-(\mathrm{BEDT}-\mathrm{TTF})_{2} \mathrm{Hg}(\mathrm{SCN})_{2} \mathrm{Cl}$ : Optical and transport studies. J. Phys. Condens. Matter 2017, 29, 055601. [CrossRef]

43. Beyer, R.; Dressel, M. Piston pressure cell for low-temperature infrared investigations. Rev. Sci. Instr. 2015, 86, 053904. [CrossRef] 
44. Rösslhuber, R.; Uykur, E.; Dressel, M. Pressure cell for radio-frequency dielectric measurements at low temperatures. Rev. Sci. Instr. 2018, 89, 054708. [CrossRef] [PubMed]

45. Shibauchi, T.; Carrington, A.; Matsuda, Y. A Quantum Critical Point Lying Beneath the Superconducting Dome in Iron Pnictides. Ann. Rev. Condens. Matter Phys. 2014, 5, 113-135. [CrossRef]

46. Zapf, S.; Dressel, M. Europium-based iron pnictides: A unique laboratory for magnetism, superconductivity and structural effects. Rep. Progr. Phys. 2016, 80, 016501. [CrossRef]

47. Abeles, B.; Cohen, R.W.; Cullen, G.W. Enhancement of Superconductivity in Metal Films. Phys. Rev. Lett. 1966, 17, 632-634. [CrossRef]

48. Cohen, R.W.; Abeles, B. Superconductivity in Granular Aluminum Films. Phys. Rev. 1968, 168, 444-450. [CrossRef]

49. Deutscher, G.; Gershenson, M.; Grünbaum, E.; Imry, Y. Granular Superconducting Films. J. Vac. Sci. Technol. 1973, 10, 697-701. [CrossRef]

50. Pracht, U.S.; Bachar, N.; Benfatto, L.; Deutscher, G.; Farber, E.; Dressel, M.; Scheffler, M. Enhanced Cooper pairing versus suppressed phase coherence shaping the superconducting dome in coupled aluminum nanograins. Phys. Rev. B 2016, 93, 100503. [CrossRef]

51. Koonce, C.S.; Cohen, M.L.; Schooley, J.F.; Hosler, W.R.; Pfeiffer, E.R. Superconducting Transition Temperatures of Semiconducting $\mathrm{SrTiO}_{3}$. Phys. Rev. 1967, 163, 380-390. [CrossRef]

52. Binnig, G.; Baratoff, A.; Hoenig, H.E.; Bednorz, J.G. Two-Band Superconductivity in Nb-Doped SrTiO3. Phys. Rev. Lett. 1980, 45, 1352-1355. [CrossRef]

53. Van der Marel, D.; van Mechelen, J.L.M.; Mazin, I.I. Common Fermi liquid origin of $T^{2}$ resistivity and superconductivity in n-type $\mathrm{SrTiO}_{3}$. Phys. Rev. B 2011, 84, 205111. [CrossRef]

54. Thiemann, M.; Beutel, M.H.; Dressel, M.; Lee-Hone, N.R.; Broun, D.M.; Fillis-Tsirakis, E.; Boschker, H.; Mannhart, J.; Scheffler, M. Single-Gap Superconductivity and Dome of Superfluid Density in Nb-Doped SrTiO 3 . Phys. Rev. Lett. 2018, $120,237002$. [CrossRef] [PubMed]

55. Collignon, C.; Lin, X.; Rischau, C.W.; Fauqué, B.; Behnia, K. Metallicity and Superconductivity in Doped Strontium Titanate. Ann. Rev. Condens. Matter Phys. 2019, 10, 25-44. [CrossRef]

56. Gastiasoro, M.N.; Ruhman, J.; Fernandes, R.M. Superconductivity in dilute SrTiO 3 : A review. Ann. Phys. 2020, 417, 168107. [CrossRef]

57. Ye, J.T.; Zhang, Y.J.; Akashi, R.; Bahramy, M.S.; Arita, R.; Iwasa, Y. Superconducting Dome in a Gate-Tuned Band Insulator. Science 2012, 338, 1193-1196. [CrossRef] [PubMed]

58. Lu, J.; Zheliuk, O.; Chen, Q.; Leermakers, I.; Hussey, N.E.; Zeitler, U.; Ye, J. Full superconducting dome of strong Ising protection in gated monolayer $\mathrm{WS}_{2}$. Proc. Natl. Acad. Sci. USA 2018, 115, 3551-3556. [CrossRef]

59. Saito, Y.; Ge, J.; Watanabe, K.; Taniguchi, T.; Young, A.F. Independent superconductors and correlated insulators in twisted bilayer graphene. Nat. Phys. 2020, 16, 926-930. [CrossRef]

60. Takabayashi, Y.; Prassides, K. Unconventional high- $T_{\mathcal{c}}$ superconductivity in fullerides. Phil. Trans. R. Soc. A 2016, 374, 20150320. [CrossRef]

61. Takabayashi, Y.; Ganin, A.Y.; Jeglič, P.; Arčon, D.; Takano, T.; Iwasa, Y.; Ohishi, Y.; Takata, M.; Takeshita, N.; Prassides, K.; et al. The Disorder-Free Non-BCS Superconductor $\mathrm{Cs}_{3} \mathrm{C}_{60}$ Emerges from an Antiferromagnetic Insulator Parent State. Science 2009, 323, 1585-1590. [CrossRef]

62. Ganin, A.Y.; Takabayashi, Y.; Jeglič, P.; Arčon, D.; Potočnik, A.; Baker, P.J.; Ohishi, Y.; McDonald, M.T.; Tzirakis, M.D.; McLennan, A.; et al. Polymorphism control of superconductivity and magnetism in $\mathrm{Cs}_{3} \mathrm{C}_{60}$ close to the Mott transition. Nature 2010, 466, 221-225. [CrossRef]

63. Ardavan, A.; Brown, S.; Kagoshima, S.; Kanoda, K.; Kuroki, K.; Mori, H.; Ogata, M.; Uji, S.; Wosnitza, J. Recent Topics of Organic Superconductors. J. Phys. Soc. Jpn. 2012, 81, 011004. [CrossRef]

64. Gati, E.; Tutsch, U.; Naji, A.; Garst, M.; Köhler, S.; Schubert, H.; Sasaki, T.; Lang, M. Effects of Disorder on the Pressure-Induced Mott Transition in $\kappa$-(BEDT-TTF $)_{2} \mathrm{Cu}\left[\mathrm{N}(\mathrm{CN})_{2}\right] \mathrm{Cl}$. Crystals 2018, 8, 38. [CrossRef]

65. Furukawa, T.; Miyagawa, K.; Itou, T.; Ito, M.; Taniguchi, H.; Saito, M.; Iguchi, S.; Sasaki, T.; Kanoda, K. Quantum Spin Liquid Emerging from Antiferromagnetic Order by Introducing Disorder. Phys. Rev. Lett. 2015, 115, 077001. [CrossRef] [PubMed] 